\title{
Los ejemplares de Freud
}

\section{The exemplars of Freud}

\author{
LETICIA O. MINHOT \\ Universidad Nacional de Córdoba, Argentina | UNC
}

\begin{abstract}
RESUMEN Este trabajo se propone, a partir de la lectura que Rouse hace de Kuhn, considerar los casos que Freud presenta a lo largo de su obra bajo la hipótesis de que los mismos constituyen prototipos de casos con la finalidad de constituir un dominio de un campo científico. Esta lectura se aparta de la lectura tradicional y enfatiza el rol central que Kuhn le otorga a los ejemplares en el seno de una matriz disciplinar, dándole supremacía a la práctica con respecto al momento teórico. Mientras la lectura tradicional considera las matrices como holismos teóricos, Rouse las percibe como holismos de prácticas. Estos casos son considerados como ejemplares de la matriz disciplinar freudiana. A este énfasis le sumamos la consideración de Nickles para quien los ejemplares deben ser valorados como prototipos con poder generativo. Se examinan los rasgos de dos grupos de ejemplares, un primer grupo de ejemplares de situaciones traumáticas y un segundo grupo que pertenecen al psicoanálisis propiamente dicho. Se espera que la comparación entre ambos aporte elementos para sostener la presencia de dos matrices disciplinares en la obra de Freud.
\end{abstract}

Palabras clave holismo de prácticas - ejemplares - prototipos - trauma - psicoanálisis.

\begin{abstract}
This work is proposed on the basis of reading which Rouse makes of Kuhn. The cases presented by Freud throughout his work are considered as exemplars within a matrix. This reading was a departure from the traditional reading and emphasizes the central role that Kuhn gives exemplars in disciplinary matrix. It gives supremacy in practice with regard to the theoretical time. While traditional reading considers the matrix as theoretical holism, Rouse perceives as holism of practices. In addition, this work considers the generative power of the exemplary as idealized prototype. There are two sets of exemplars, the first group of exemplars of traumatic situations and a second group that belong to the psychoanalysis proper said. It is expected that the comparison between the two groups will show the presence of two disciplinary matrices in Freud's work.
\end{abstract}

Key words holism of practice - exemplars - prototypes - trauma - psychoanalysis.

\section{Introducción}

Freud presenta a lo largo de su obra una serie de casos. ¿Cuál es el papel que éstos cumplen? Ellos no están allí con la finalidad de verificar sus teorías, tampoco están para mostrar el éxito de la terapia. Algunos ni siquiera fueron pacientes. Tomados en conjunto, ipodríamos decir que ellos constituyen una única serie? En este trabajo estos casos son tomados como ejemplares paradigmáticos. Ellos están para dar visibilidad a modos de tratar con los síntomas. Son presentados en dos grupos, el primero de ejemplares de situaciones traumáticas - conjunto de historiales clínicos que datan del período que va de 1889 a 1892 - y un segundo grupo que pertenecen al psicoanálisis 
propiamente dicho - pertenecientes a la época de la primera tópica desde 1900 a 1920. Se espera que la comparación entre ambos grupos permita mostrar la presencia de dos matrices disciplinares en la obra de Freud. La periodización y la presencia de dos series de casos se asocian a cambios teóricos y clínicos-terapéuticos que tuvieron lugar en su obra. El segundo grupo es mencionado como "propiamente psicoanalíticos" porque el propio Freud considera que el psicoanálisis surge cuando abandona la teoría de la seducción. Este cambio teórico es acompañado de un cambio de terapia, del método catártico pasa al interpretativo. De la pretensión de eliminar el síntoma por anular su causa se pasa a la de elucidarlo. Así, las dos series de ejemplares se corresponden con modelos ontológicos y heurísticos diferentes, con distintas generalizaciones guías y distintas terapias. No obstante, la visibilidad se logra a través de los ejemplares.

Rouse presenta, frente a la lectura más tradicional, un Kuhn radical, efectivamente revolucionario. Esta perspectiva se aparta de la concepción predominante - Rouse se refiere a esta como el "Kuhn de los filósofos" - y, enfatizando el rol central que el propio Kuhn le otorga a los ejemplares en el seno de una matriz disciplinar, le otorga supremacía a la práctica con respecto al momento teórico. Mientras la lectura tradicional considera las matrices como holismos teóricos, Rouse las percibe como holismos de prácticas. Este cambio de holismo implica una modificación en lo que se entiende por "significado". Tomando la diferencia planteada por Taylor entre "significado" como concepto hermenéutico y "significado" como concepto lingüístico, se aborda la separación entre ambos tipos de holismos y la visión de ciencia que cada uno conlleva. Desde la perspectiva de Rouse, la reconstrucción de las teorías debe focalizar en los ejemplares pues ellos ofrecen el dominio empírico de la misma. Aquí, su estudio nos permite identificar los componentes teóricos y el lenguaje desde los que fueron estructurados, las perspectivas metafísicas que encarnan como asimismo los valores epistemológicos y culturales, las técnicas que se emplean, entre otras, para establecer la anamnesis de un caso. Respecto a estas últimas, se pueden distinguir dos momentos. Un primer momento en que se presentan los datos empíricos sin establecer nexos entre ellos. Ahora bien, sólo podemos hablar con propiedad de que estamos ante un caso cuando el terapeuta reconstruye, en un segundo momento, los nexos. Cuando Freud habla de la técnica para establecer la anamnesis se refiere a este segundo momento. Desde la lectura de Rouse, la técnica es ella misma una interpretación no reductible a procedimientos regulados por reglas. Se espera que este trabajo pueda aportar elementos para identificar estas comprensiones ancladas en estas técnicas.

Se ha escrito mucho respecto a los casos de Freud, por ejemplo, respecto al Hombre de los Lobos se lo puso en tensión con la propia versión de Sergei Pankejeff (nombre real del Hombre de los Lobos) según la cual él rechaza la interpretación de Freud. ${ }^{2}$. Sin embargo, en realidad, estas lecturas críticas de los casos dirigidas a su coincidencia con la propia perspectiva de las personas que protagonizan los casos no ven a los mismos como ejemplares. El Hombre de los Lobos como prototipo no es el individuo Sergei Pankejeff. En este trabajo los casos son presentados como ejemplares, es decir como estructuras con potencial generativo para establecer relaciones de similitudes. No se va a mostrar cómo la práctica concreta del psicoanálisis resuelve problemas a partir de estos casos arquetípicos pues esto superaría en mucho los objetivos de este trabajo. Sólo se pretende presentar los prototipos a partir de los cuales se generaría una comunidad científica.

\section{Modos de leer a Kuhn}

¿En qué consistió la revolución Kuhniana? En 1991, George A. Reisch se planteó la pregunta si ¿Mató Kuhn al empirismo lógico? Para este autor, si bien es cierto que La estructura de las revoluciones científicas tuvo una considerable percusión en la concepción de la ciencia del positivismo lógico, eso no significa la ausencia de semejanzas entre Kuhn y Carnap. ${ }^{2}$ Este libro de Kuhn no contiene una refutación del empirismo lógico sino, más bien, presenta una concepción del cambio científico revolucionario que es similar a la de Carnap. Más tarde, el artículo de Gürol Irzik y Teo Grünberg de 1995 analiza las semejanzas entre las visiones de la ciencia de Kuhn y Carnap. Aquí, los autores consideran errónea la 
perspectiva que sostiene que hay una revolución radical entre Kuhn y el positivismo lógico. ${ }^{3}$ Sin embargo, más allá de estas potenciales similitudes, lo real es que La estructura de las revoluciones científicas fue un texto que fue percibido como revolucionario.

Taylor distingue entre el "significado" como concepto hermenéutico y como lingüístico, tenemos que el primero se aplica a cosas, acciones, situaciones y el segundo, sólo a enunciados o términos. El significado hermenéutico tiene una estructura de tres dimensiones: es el significado de algo para un sujeto dado o para una comunidad y se da en relación con otros significados. El significado lingüístico, en cambio, es también un significado de algo para un sujeto y existe en un campo de significados, pero, además, liga un significante y un referente. Este último es una estructura de cuatro dimensiones. ${ }^{4}$ Rouse distingue dos Kuhn. El Kuhn de los filósofos, - básicamente el Kuhn de Israel Schefler, Dudley Shapere, Frederick Suppe, entre otros - y el Kuhn radical que él propone. El Kuhn de los filósofos trata los problemas relativos derivados de mundos diferentes - como el de inconmensurabilidad, por ejemplo - desde el concepto lingüístico de significado. La ciencia es una actividad que tiene por objetivo construir teorías que representan al mundo, donde "mundo" es la percepción y descripción de ciertos fenómenos, de una manera determinada, por sistemas lingüístico-conceptuales. Es central a esta concepción la problemática derivada de los vínculos entre las teorías y aquello a lo que éstas se refieren. Es decir, a la cuarta dimensión que caracteriza al sentido lingüístico de "significado". Si bien, en autores como Kuhn y Quine, no hay un lenguaje observacional teóricamente neutro y el significado de los términos científicos está dado por su lugar en redes teóricas, esto es, la referencia está generada por las redes teóricas. Los significados (lingüísticos) se derivan de un holismo teórico. ${ }^{5}$ Por ello, las matrices disciplinares son inconmensurables pues los términos tienen distintos significados - refieren a cosas diferentes - al estar en redes teóricas disimiles.

Así, por ejemplo, el Kuhn de Frederick Suppe es presentado de la siguiente forma: las formulaciones del último Wittgenstein sobre el lenguaje y la psicología filosófica jugaron un papel importante en las concepciones de Toulmin y de Hanson. Se comenzaba a abrir una noción de ciencia que la consideraba una empresa social y la epistemología debía considerar la dinámica del desarrollo teórico. Para esta concepción, la ciencia se hace desde sistemas lingüísticos conceptuales determinados que configuran conceptualmente la forma de experimentar el mundo.

En suma, la ciencia se hace desde una weltanschauung o Lebenswelt, y la tarea de la filosofía de la ciencia consiste en analizar lo característico de las weltanschauung científicas, lo característico de los sistemas lingüístico-conceptuales desde los que se hace la ciencia. Las teorías se interpretan en términos de una weltanschauung; de ahí que para comprenderlas sea necesario comprender la weltanschauung. ${ }^{6}$

La visión del mundo - es decir, sistemas lingüístico-conceptuales - es una perspectiva conceptual desde la que se prescribe y proscribe creencias, determinando los hechos de los que se debe ocupar la ciencia. La weltanschauung es un conjunto de creencias compartidas. La weltanschauung kuhniana se acerca al holismo teórico de Quine, una red de creencias y valores. Es el lenguaje que los científicos hablan en un momento dado. Los científicos viven en un mundo diferente luego de una revolución porque viven en sistemas lingüísticos-conceptuales diferentes. Este Kuhn toma "significado" en su sentido lingüístico, nuestros compromisos ontológicos están establecidos por la weltanschauung en que vivimos, esto es, los referentes son fijados por la gran teoría total. El significado sigue requiriendo de este enlace de un significante con un referente. La diferencia con Quine reside en cómo se posiciona este Kuhn con respecto a la interpretación. Mientras en Quine la interpretación está restringida a la traducción, en Kuhn la interpretación comienza a trabajar en los límites de la traducción, en particular, en aquellas circunstancias que él considera inconmensurables, esto es, cuando encuentra términos en un lenguaje diferente al suyo para los que no puede establecer los referentes con los términos de su propio lenguaje. La interpretación implica que se ha aprendido el lenguaje. Es posible, de esta manera, aprender un lenguaje aunque no se hayan traducido muchos de sus términos. En Quine, el significado lingüístico de un término que pertenece a otro lenguaje se alcanza determinando la referencia por medio de un manual de traducción, en Kuhn, tal significado lingüístico se alcanza determinando la referencia, lo cual es posible si se hace nativo y aprende el lenguaje. Kuhn, en tanto historiador de la ciencia reconoce que lo importante es hacerse nativo y poder interpretar y no la traducción. Lo que es importante destacar aquí, es que en este Kuhn la interpretación está orientada a creencias. 
Interpretar una weltanschauung diferente es comprender la teoría total de la que se era extranjero. El cambio gestáltico es un cambio de weltanschauung que instala al intérprete en otro mundo, en otro sistema lingüístico-conceptual, en otra gran teoría.

Frente a este Kuhn, Rouse propone uno radical. Las tesis que lo caracterizan y diferencian del Kuhn antes expuesto son las siguientes: 1) la investigación científica presupone una comprensión de aquello con lo cual se está tratando que no puede ser explícito en las formas de representaciones teóricas, es la comprensión que suponen las habilidades de los científicos, sus técnicas y proyectos; 2) el lenguaje sólo es preciso en un sentido estrecho, el lenguaje que los científicos emplean en sus habilidades está encarnado en su uso y referido a situaciones concretas y no es completamente articulado; 3 ) las actividades de los científicos son interpretaciones de lo que es asumido en la práctica cotidiana de la investigación, esto es, holismo de las prácticas; 4) los científicos normalmente no prueban sus teorías, las usan. ${ }^{7}$ Cabe aclarar que el propio Kuhn prefirió optar, en escritos posteriores al '69, por la perspectiva anterior, más cercana a la del de los filósofos, esto es, una perspectiva más dominante en la filosofía de la ciencia - en particular en el significado entendido como significado lingüístico. Sin embargo, el Kuhn de los filósofos no es un Kuhn revolucionario, tal como sostienen, entre otros, los artículos señalados al comienzo de este trabajo.

Desde esta lectura radical, las matrices disciplinares no se fundan en acuerdos basados en compromisos teóricos caracterizados por la creencia compartida en enunciados. Vivir en una matriz disciplinar es adquirir y aplicar habilidades - aplicar conceptos, emplear técnicas matemáticas y de laboratorio - especificadas por modos ejemplares de conceptuar e intervenir en contextos empíricos particulares. Para el Kuhn radical la ciencia normal envuelve prácticas compartidas, no creencias compartidas. El cambio de un paradigma a otro no es una conversión a nuevas creencias sino una conversión a una nueva forma de vida.

Se abandona en esta perspectiva radical la idea del lenguaje como discurso sobre el mundo. Lo nuevo es la práctica con su propio modo de comprender, no reductible a representación teórica. Los significados ahora también dependen de su ubicación en una red, pero lo que ha variado es la red, ya no envuelve solamente conceptos, creencias, sino también técnicas, instrumentos y habilidades. Se tiene así otro tipo de holismo: holismo de las prácticas. Aquí, interpretar no es formar hipótesis sobre el mundo, sino actuar de una determinada manera. En el holismo teórico la "teoría total" es un campo de enunciados y la inconmensurabilidad es un problema entre campos de enunciados. En el holismo de la práctica, en cambio, la totalidad contiene prácticas, - el lenguaje entre ellas. Vivir en mundos diferentes es solucionar problemas de modo diferente. En este sentido, la interpretación del mundo de una determinada matriz disciplinar sólo se elucida por la interpretación de las prácticas. Lo inconmensurable, desde este holismo, se sitúa entre campos que contienen modos diferentes de resolver problemas porque diferentes "formas de vida" constituyen sus campos de posibilidades. El Kuhn radical adhiere así a la interpretación como comprensión pero, a diferencia del Kuhn apropiado por la tradición, los significados envueltos son significados hermenéuticos, no significados lingüísticos. La interpretación es el resultado de estar socializado en prácticas que son interpretaciones accesibles públicamente en el contexto del mundo social. La accesibilidad pública es el criterio que legitima una interpretación.

Desde esta perspectiva, las interpretaciones que constituyen un mundo no están completa ni necesariamente presentes en estados mentales. Si bien pueden expresarse en palabras, no está originalmente articulada de este modo. No se trata de interpretación de prácticas dadas sino de que cada práctica es una interpretación. Esta comprensión no emerge de un conjunto de creencias sino que está incorporada en el hacer y, como trasfondo, es el mundo que una determinada comunidad científica habita. Las prácticas no surgen ni de creencias ni de reglas, por lo que no se trata de hacerlas explícitas sino de interpretar las interpretaciones que están contenidas en las prácticas.

Este trabajo adhiere a esta lectura de Rouse. Ahora bien, de los sentidos de "paradigma" caracterizados como elementos de la matriz disciplinar ¿cuál es el verdaderamente revolucionario?, es decir ¿cuál es el que respalda mejor al Kuhn radical de Rouse?

Quizás uno de los aportes más importantes de Kuhn reside en su enfoque sobre los aspectos metodológicos de la investigación científica: la práctica científica está centrada en la resolución de problemas basada en ejemplares o casos. 
Cuando Kuhn sustituye el concepto de paradigma por el de matriz disciplinar, los ejemplares son el cuarto componente y el elemento central de una matriz. Los ejemplares son

Las concretas soluciones de problemas que, empleadas como modelos o ejemplos, pueden reemplazar reglas explícitas como base de la solución de los restantes problemas de la ciencia normal (Kuhn, 1969: 269). ${ }^{8}$

Los ejemplares sirven como modelos para resolver futuros problemas. El éxito de un ejemplar atraerá el apoyo de los científicos y un campo de la ciencia puede ser definido por los ejemplares que éste emplea. La participación en un campo científico necesariamente los involucra. Para Kuhn, las relaciones de similitud adquiridas a partir de un casopatrón tienen prioridad con respecto a las derivaciones de primeros principios a través de reglas. Estas últimas jugaron un rol central en la filosofía de la ciencia dominante previa a Kuhn. Nickles ${ }^{9}(1998,2000)$ sostiene que la investigación kuhniana puede ser reconstruida como razonamientos basados en casos en oposición a razonamientos basados en reglas. Los primeros no resuelven cada problema desde cero sino que hacen coincidir nuevos casos a viejos y adaptan sus soluciones. El conjunto de ejemplares de un dominio junto con las relaciones de similitud adquiridas representan la experiencia científica de ese dominio. Las revoluciones producen nuevos ejemplares y el complejo de similitudes cambia. El conocimiento se basa en aprender estas similitudes porque ello supone adquirir una forma de ver, de ahí la centralidad de los ejemplares en la matriz disciplinar. Las generalizaciones funcionan cuando se asocian con estos patrones de soluciones, porque son ejemplos concretos del comportamiento de la ley-esquema en la realidad. Pero este aprender a ver las similitudes no es algo que se haga a través de reglas, sino que se adquiere a través de la práctica científica misma y es previa, incluso, a cualquier criterio de analogía. La base de este conocimiento es la interpretación y no la percepción. Ser capaz de recorrer esa horizontalidad es adquirir una forma de ver.

Una de las técnicas fundamentales por las que los miembros de un grupo, ya sea toda una cultura o una subcomunidad de especialistas dentro de ella, aprenden a ver las mismas cosas cuando se encuentran ante los mismos estímulos, es al verse ante ejemplos de situaciones que sus predecesores en el mismo grupo ya habían aprendido a ver como similares y como diferentes de otras especies de situaciones. ${ }^{10}$ (Kuhn, 1969: 296)

Ese aprender a ver responde a un proceso interpretativo y no a un proceso perceptivo. A través de los ejemplos es que se aprende a "ver" entidades teóricas. Ver, no en el sentido de percepción, sino de interpretación. Este ver no es el resultado de reglas de aplicación sino de la práctica, que se basa - partiendo de los ejemplares que aparecen en el texto - en la similitud. Los ejemplares fijan una interpretación cuando, a través de ellos, se ven otras situaciones como casos de las mismas entidades teóricas. Como la similitud no es aprendida por criterios de similitud, no hay criterios que determinen una interpretación: éstas se justifican por un triunfo histórico. Esa separación, entre interpretación y criterios, se refleja en la distancia que coloca, por un lado, leyes, esquemas y, por el otro, ejemplares. Esto indica la no deductividad de los segundos a partir de los primeros, los cuales, no funcionan como criterios de similitud, ni su aplicación a los ejemplares se deriva deductivamente. La interpretación depende de la práctica. La presencia de casospatrón es la base para la aceptación de cualquier teoría y para la enseñanza de ella, pues adiestra para poder ver las entidades que introduce.

Los ejemplares constituyen un conjunto base que le da a la comunidad científica los cimientos para resolver un número potencialmente infinito de problemas concretos como asimismo las soluciones admisibles, en ello radica su productividad. Estos ejemplos compartidos de soluciones a problemas técnicos ilustran las generalizaciones simbólicas, le dan el contenido empírico a esos componentes formales. En la práctica, cuando se va a aplicar una determinada generalización simbólica, hay toda una variedad de situaciones para los que el esbozo de ley debe adquirir la forma que permite la interrelación entre ellas. El procedimiento por el que se aplican las leyes no es sólo sintáctico, puesto que se trata de darles un contenido empírico. Esta interrelación se basa en la analogía entre las distintas situaciones y, por ello, la fórmula puede aplicarse tanto en una como en otra. La aplicabilidad no sigue un sentido vertical -de la fórmula a 
los casos-, sino que recorre una horizontalidad entre casos análogos. Se trata de algo que se aprende con la práctica. En esta analogía se fundamenta la función de los ejemplares como modelos de soluciones.

Los científicos resuelven los enigmas modelándolos sobre anteriores soluciones de enigmas, a menudo recurriendo apenas a las generalizaciones simbólicas. (Kuhn, 1969: 290). ${ }^{11}$

No obstante, Nickles ${ }^{12}$ (1998; 2000) le critica a Kuhn que presentara a los ejemplares como logros históricos fijos, como nudos inmóviles para futuras investigaciones. Es decir, no tiene una consideración histórica, dinámica de ellos, son determinantes pasivos de la experiencia. Para Kuhn un ejemplar es una solución específica, un logro concreto en la historia de la ciencia. El reproche de Nickles va en la dirección de Kuhn no haber aclarado lo suficiente que, para cumplir la función de ejemplar, ese logro concreto luego es esquematizado para poder ser empleado por las generaciones sucesivas en diferentes situaciones. Para que un caso logre su estatus de ejemplar se requiere de la amplia aceptación de la comunidad científica para que sea tomado como base para su futuro trabajo, de esta forma se transforma en ejemplar y adquiere una dimensión normativa y transmite objetivos y estándares. Se torna en heurística construida sobre las bases de la práctica real de solucionar problemas y se vincula con estrategias familiares de resolución de problemas. Cuando esto sucede se ha idealizado por diferentes procesos de abstracción. Para Nickles ${ }^{13}$ (1998; 2000) los ejemplares son guías positivas para la investigación en marcha, lejos de ser pasivos son generativos pues contienen un dominio infinito de soluciones. Para ser generativos se requiere que dejen de ser casos individuales para transformarse en prototipos. En esta línea, es posible avanzar en una consideración dinámica de los ejemplares y entrever mejor el alcance de los mismos como una condición necesaria para la constitución de una comunidad científica.

\section{Los ejemplares de situaciones traumáticas}

En este apartado, el propósito consiste en mostrar los modos ejemplares de intervenir en contextos empíricos particulares que consideran situaciones traumáticas. Los ejemplares son los siguientes casos: Emmy, ${ }^{14}$ Lucy, ${ }_{1}^{15}$ Catarina ${ }^{16}$ y Elizabeth. ${ }^{17}$

Éstos son un conjunto de historiales clínicos que datan del período que va de 1889 a 1892 y que Freud trabajara conjuntamente con Breuer. ${ }^{18}$ Los pacientes son mujeres cuyos diagnósticos son diferentes tipos de histerias. La fórmula etiológica que aquí se aplica es simple: hechos reales - vivencias efectivas - traumáticos en los que la reacción normal fue reprimida, dando lugar, posteriormente a un síntoma. Éstos constituyen una solución exitosa para el problema de establecer la relación entre síntomas y el recuerdo de un momento traumático. La técnica para elaborar la anamanesis de los casos se basa en la búsqueda del recuerdo traumático con la finalidad de eliminar los síntomas. En todos, el hecho traumático puede ser de diversas índoles: una tentativa de abuso sexual por parte de algún adulto en una época en la cual el individuo no comprende exactamente lo que ocurre, una humillación o presenciar objetos impresionantes - tales como cadáveres, animales asustadores etc. Lo fundamental, el hecho tuvo que despertar una reacción tal como deseo de gritar, de llorar, de huir o de devolver la afrenta y esto no fue realizado, esa reacción fue reprimida. En todos estos casos el analista siempre trata de eliminar el síntoma eliminando o tornando indefinida el recuerdo que previamente trae a la conciencia. Los síntomas son eliminados uno a uno, reconstruyendo la cadena asociativa que los liga con el hecho traumático respectivo, para enseguida tornar indefinido el propio recuerdo. La sugestión es el instrumento principal en la eliminación de la imagen mnemónica. Así se van revelando camadas de recuerdos. Al eliminar un síntoma, éste es sustituido por uno nuevo, que lleva a un hecho anterior al que provoca el síntoma ya eliminado. Eliminada la causa se elimina el efecto, es la fórmula terapéutica. Todos los síntomas somáticos de las histéricas son símbolos mnemónicos de aquello hechos dolorosos. De esta forma se va hacia la escena traumática primordial, encubierta por recuerdos sucesivos, generando cada uno un síntoma también encubridor del anterior. Cada caso de este grupo presenta muchos ejemplos de este tipo. Para cada síntoma se trata de hechos en los cuales el síntoma encuentra su fuerza. El síntoma, 
a diferencia como será visto desde la segunda etiología, no es la manifestación de un deseo, sino es un símbolo del recuerdo doloroso. Cuando entra el elemento sexual en la etiología, como en el caso Catarina, entra como traumático en un momento en que no se comprende lo que sucede. Varios de los casos muestran cómo un hecho reaviva el recuerdo de otros hechos análogos, transformándose en símbolo mnemónico.19

El cambio de visión de los síntomas implicará un cambio de terapia y de técnica de elaboración de la anamnesis de los casos. En el método interpretativo estos no serán eliminados mediante la eliminación del recuerdo, porque lo patógeno ya no se constituye desde y por reminiscencias.

\section{Los ejemplares del psicoanálisis}

En este punto enfatizo el análisis de un caso del período de la primera tópica para mostrar un modo ejemplar de intervenir en contextos empíricos propiamente psicoanalíticos. ${ }^{20}$ Es decir, cuando ya Freud abandonó la teoría de la seducción.

La técnica para la elaboración de la anamnesis de los casos de esta serie debe reconstruir algunas escenas, aquellas que no están presentes en la conciencia del paciente. A partir de ellas, capas más profundas del inconsciente se van develando y llega tan lejos cuanto le permitió la duración del tratamiento. Los ejemplares de la etapa psicoanalítica relativos a la primera tópica - Dora, Juanito, Schreber, El Hombre de las Ratas, El Hombre de los Lobos - muestran el regreso a un punto de fijación en el desarrollo de la libido que pertenece a la actividad sexual infantil, la cual por ser perversa genera rechazo y mueve las fuerzas de la represión. Según sea el caso de la patología, esta fórmula adquiere una versión especial la cual depende del punto de fijación. En la progresión cronológica de los casos hay un abandono significativo de las vivencias con valor etiológico. Cada vez más, ellas tienen, meramente, la función de causas accidentales, frustraciones actuales, no del pasado, que desencadenan lo que estaba predispuesto, esto es, la regresión a puntos de fijación. A medida que ese punto de fijación se va constituyendo según el complejo paterno, podemos hablar de una solución del enigma que presentan las patologías a través del complejo de Edipo, esto significa una solución en términos de retorno a objetos libidinales de la infancia primera. Vemos así una progresión en el planteo de los casos que va desde la figura paterna a un complejo paterno, del que el complejo de castración es una parte. Ese complejo paterno es el de Edipo, el cual alcanza su estatus de esquema paradigmático en el caso del Hombre de los Lobos. Los casos anteriores son preparaciones para la aplicación de este esquema, pues para que éste funcione es necesario concebir el desarrollo de la libido a través de organizaciones y de una realidad psíquica que resulta de ese desarrollo. Tales organizaciones sólo son analizadas en este caso. El complejo de Edipo no se refiere al padre efectivo sino a un esquema que determina una escena en la que tiene lugar un conjunto de representaciones. Resolver los casos a través de este complejo paterno no significa reconocer en éste la etiología de la patología que representan. Resolver los casos a través de este complejo significa reconocer la fuerza de la represión que lo mantiene en el inconsciente o, lo que es lo mismo, reconocer los afectos primarios que fueron sofocados, pero no eliminados, y que se manifiestan en los síntomas. Una de las diferencias fundamentales con la etiología anterior y, por lo tanto, con los ejemplares antes referidos, es relativa a lo sofocado. Allá se sofocaban reacciones a vivencias desagradables, aquí se sofocan afectos primarios. Lo que sale a luz en estos ejemplares es la relación entre la frustración actual que se enlaza con una fuerza del pasado generando un conflicto. Cada caso es un esfuerzo por dar contenido empírico a estos elementos. Si comparamos los casos que Freud presenta cuando adhiere a la teoría de la seducción con los casos que analizamos a continuación se revela que, en aquellos, se aprende a ver hechos del pasado que provocan estados presentes, mientras que, en éstos, se ve frustraciones presentes que llevan al pasado.

Si se focaliza, por ejemplo, en el Hombre de los Lobos, el resumen cronológico que se dispone de este caso no es de Strachey como sucede en Dora, Juanito, Schreber y El Hombre de las Ratas. Es del propio Freud, en nota agregada en $1924 .{ }^{21}$ Resulta importante comparar con los resúmenes anteriores, ahora no se dispone sólo de un 
conjunto de datos de la vida y entorno del paciente, sino que se está ante una reconstrucción. Basta con percibir la presencia del hecho ocurrido durante el padecimiento de malaria del niño. La observación del coito de los padres no es un dato como los otros, sino que es una escena que Freud reconstruye, es una escena inferida de la interpretación de un sueño.

Me gustaría mucho saber si la escena primordial fue en mi paciente fantasía o vivencia real, pero remitiéndose a otros casos parecidos es preciso decir que en verdad no es muy importante decidirlo. ${ }^{22}$

Es en ese hecho en el que se apoya toda la edificación del caso, porque el ejemplar está, justamente, mostrando la determinación psíquica de los síntomas, la escena primordial interesa por el modo en que se entrama en la realidad psíquica y no si efectivamente sucedió o no. Esta es la primera gran diferencia con los ejemplares que mostraban los traumas del apartado anterior. En aquellos, los recuerdos eran de hechos efectivos, externos a la psiquis del individuo. La escena primordial le da sentido a los vínculos de los contenidos manifiestos y latentes que el caso plantea. No obstante, esta escena tan fundamental no es deducida lógicamente del sueño, sino que es dada a priori.

La enfermedad, en su forma definitiva, que lleva al joven a pasar por diferentes hospitales es diagnosticada por los psiquiatras como insanía maníaco depresiva. El diagnóstico de Freud es el de secuela de una neurosis obsesiva. ${ }^{23}$ Este caso no es la presentación de la patología en su forma acabada, sino que es un caso de una neurosis infantil desde el recuerdo del adulto. La enfermedad, el tratamiento y la cura, no aparecen en la descripción. Lo que el caso quiere mostrar es la sexualidad infantil y el rol de las fantasías en la formación de los síntomas, de ese modo es un ejemplar que intenta solucionar el esclarecimiento del mecanismo de formación de síntomas en la neurosis obsesiva. ${ }^{24}$

En el tratamiento de este caso Freud se sirve de un sueño de angustia, el sueño de los lobos, de la interpretación de éste se derivan los supuestos sustanciales al esclarecimiento de la formación de los síntomas.

He soñado que es de noche y estoy en mi cama. (Mi cama tiene los pies hacia la ventana, frente a la ventana había una hilera de viejos nogales. Sé que era invierno cuando soñé y de noche.) De repente, la ventana se abre sola y veo con gran terror que sobre el nogal grande frente a la ventana están sentados unos cuantos lobos blancos. Eran seis o siete. Los lobos eran totalmente blancos y parecían más bien con unos zorros o perros ovejeros, pues tenían grandes rabos como zorros y sus orejas tiesas como de perros al acecho. Presa de gran angustia, evidentemente de ser devorado por los lobos, rompo a gritar y despierto. ${ }^{25}$

Este sueño es importante porque parece ser el primero de angustia. La interpretación del sueño lleva varios años y sólo en el tiempo próximo a la cura es que se llega a comprenderlo completamente.

El sentimiento de realidad del sueño revela que el sueño se refiere a un hecho que ocurrió de verdad y no que fue fantaseado ${ }^{26}$ - se basa en lo que había sostenido en La interpretación de los sueños. Los juicios que enfatizan la relación entre sueño y realidad pertenecen al contenido latente del sueño e indican que lo que el sueño cuenta efectivamente ocurrió. ${ }^{27} \mathrm{Si}$ el referente del sueño es un hecho que se ha olvidado, éste debió darse a una edad muy temprana. Este argumento le lleva a sostener que el sueño convoca una imagen en la que se fundamenta la interpretación. La elucidación del sueño que ofrece Freud es la siguiente:

Es de noche: es la desfiguración de "Yo había dormido".

Estoy en mi cama: comienzo de la reproducción de la escena primordial.

Sé que era invierno cuando soñé y de noche: no pertenece al contenido del sueño, sino a su recuerdo.

De repente, la ventana se abre sola: equivale a "de repente me despierto solo", con esto el resto del contenido del sueño se coloca en el presente.

El nogal grande frente: reemplaza al árbol de Navidad. El árbol alto es símbolo de voyeurismo. 
Lobos: Su número: Eran seis o siete: influencia del cuento de los siete cabritos. La desfiguración onírica cambia el número dos de la escena primordial por el de seis o siete.

Sentados sobre el árbol: sustituyen a los regalos de Navidad que se colocan en los árboles navideños.

Los lobos eran totalmente blancos: esto procede de las ropas y sábanas blancas de la escena primordial.

Lo miraban con atención: esto llega desde la escena primordial.

Parecían más bien con unos zorros o perros ovejeros, pues tenían grandes rabos como zorros: está relacionado con la castración.

Presa de gran angustia, evidentemente de ser devorado por los lobos: la angustia se explica con la relación al cuento de los siete cabritos-hijos son devorados por el lobo-padre.

La añoranza sexual que se activa durante la noche convoca una determinada imagen, la cual tiene que anudar un deseo sexual con una represión de éste pues se trata de un sueño de angustia. La imagen debe relacionarse con el temor de la castración, temor que explica el cambio de afecto. Al deseo de que llegue pronto la noche de Navidad con sus regalos se une el deseo más profundo de ser satisfecho sexualmente por el padre y al deseo de volver a ver lo que resultara tan atrayente. La imagen tiene que haber sido la de una relación sexual de los padres, la posición que éstos adoptaron es la del hombre erguido y la mujer agachada como los animales, a esta imagen es la que Freud se refiere como escena primordial. La edad en que se la observó fue más o menos la de $1 \frac{1}{2}$ año, pero la comprensión de lo que vio es de la época del sueño, no de la observación. Esta posición se infiere de varios hechos, durante la juventud sólo consigue gozar con la postura que habrían adoptado los padres en la escena primordial pero principalmente se infiere del temor que le despiertan las figuras de lobos erguidos en posición vertical. Quizás no vio a los padres, sino al coito entre animales y luego lo traslada a los padres, pero la observación de un coito es el supuesto fuerte de la interpretación onírica. Esto es, toda la interpretación se basa en un supuesto que Freud coloca en la anamnesis del caso. En el sueño se realiza el deseo de convocar la escena primordial pero ese deseo es reprimido, por eso termina en angustia.28 Con la escena primordial activada asoció masculino a activo y femenino a pasivo. El deseo sexual del sueño tiene una meta pasiva, quiere ser poseído por el padre y es esta meta la que es reprimida, por ello la angustia se dirige al lobo erguido, esto es, al padre, es un rechazo del deseo que instala el sueño. La angustia que provoca el deseo es reemplazada por fobia al lobo, tal sustitución está basada en la asociación entre la observación de sus padres y el contenido del sueño, esto es, la postura vertical. Todos los detalles de otras historias que activan ese recuerdo son incorporados como material que reemplaza la imagen primordial.

En el momento que tiene el sueño alcanza una nueva organización sexual, desde la seducción su meta sexual era pasiva, luego regresa a la organización sádico anal con meta masoquista, es común de esta época fantasías en que es castigado. El sueño lo regresa a la organización genital y, lo que hasta ese momento eran pasivo-activo, ahora se transforma en el par femenino-masculino. Es justamente la meta femenina la que cae bajo represión, la identificación con la madre en el curso del sueño le dice que si quiere ser poseído por el padre tiene que ser castrado, eso es, justamente, lo que rechaza.

Posteriores sueños de agresión hacia su hermana y hacia la gobernanta, procesaban de diferentes formas un mismo contenido: recuerdos de fantasías referidas a la infancia que se forman, quizás, en la pubertad. A partir de la manifestación de estas reminiscencias sobrevinieron otras, las referidas a los intentos de seducción de su hermana cuando eran niños.

Al final del análisis del caso, Freud ofrece un panorama del desarrollo sexual del paciente. La primera organización sexual es la canibálica u oral, ésta se revela en la angustia de ser devorado por el lobo, pero este modo de salir a la luz es como regresión desde un momento del desarrollo de la libido posterior. En el momento de la seducción que ejerce la hermana ya ha comenzado a alcanzar una organización genital pero la hermana inhibe ese desarrollo al proporcionar una meta sexual pasiva inconciliable con la acción genital masculina. La amenaza de castración por parte de la ñaña lo hace regresar a la organización sádico anal. El erotismo anal se expresa a través de sadismo que luego se transforma 
en masoquismo. El sueño lo hace volver a la organización genital, pero este progreso es rechazado en virtud de la represión y se genera la fobia. La represión es una consecuencia de la amenaza de castración y lo que se reprime es la homosexualidad genital.

El motor de esta represión parece ser la masculinidad narcisista del genital, que entra en un conflicto, preparado desde mucho antes, con la pasividad de la meta sexual homosexual. La represión es entonces un triunfo de la masculinidad. ${ }^{29}$

Pero esto no significa que el conflicto se dé entre la masculinidad y la feminidad, no es la bisexualidad el motor de la represión sino el conflicto entre el yo y la libido. El sueño fragmenta los deseos sexuales, por un lado, en el inconsciente se alcanza la organización genital bajo la forma de homosexualidad, por otro lado, el yo rechaza esa homosexualidad formando una fobia para protegerse de la angustia ante el padre, la conciencia sólo toma noticia de la angustia ante el lobo. Corrientes sádicas y masoquistas se sobreponen a la homosexual siendo las segundas las dominantes, contra ellas el yo histérico.

Que sean el sueño y la escena primordial los elementos sobre los cuales Freud construye el caso se debe a un rasgo propio de los ejemplares de esta serie y es el de mantener toda la reconstrucción en el plano intrapsíquico. Todos estos casos, a diferencia de los anteriores - los traumáticos -, plantean problemas y soluciones en el interior de la realidad psíquica. Por eso no debe extrañarnos, ni tiene ninguna implicancia el hecho de que el caso difiera drásticamente de los recuerdos del propio Hombre de los Lobos. La principal diferencia podemos detectarla en el tratamiento que Freud hace de la hermana del paciente. Dimock ${ }^{30}$ señala que este contraste es muy marcado en las consideraciones relativas al suicidio de Anna a la edad de veintiún años. El Hombre de los Lobos ubica a este hecho en el centro de su sufrimiento y toma al dolor mismo como un testimonio de la fuerza de la presencia de Anna en su vida. En oposición, en el caso freudiano este hecho no le produjo un gran sufrimiento. No obstante, Freud necesita un signo de emoción que le permita mantener su diagnóstico del Hombre de los Lobos como neurótico y no como psicótico y de esa manera presentarlo como un caso adecuado para el tratamiento psicoanalítico. Freud encuentra, más bien, en el viaje al lugar en el que se suicida el poeta ruso el vínculo con Anna que se da a través de la identificación del padre de ella con dicho poeta. Lo que es relevante en el caso freudiano son los mecanismos psíquicos por los que el dolor del hermano por el suicidio de la hermana ha sido reprimido. Por eso, para Freud, es relevante la dinámica psíquica de los primeros ocho años de vida del Hombre de los Lobos. El caso es claramente un prototipo y no puede, ni debe, identificarse con el hombre real ni es lícito exigirle a un prototipo, resultado de un proceso de idealización y abstracción, el rango completo de la experiencia humana. Es claro que un único sueño a la edad de cuatro años y una escena originaria al año y medio no pueden agotar dicho rango. Es justamente en este punto donde se difiere con Dimock, ${ }^{31}$ para este autor es criticable que Freud haya montado el caso sobre estos dos hechos solamente. La posición, aquí, consiste en valorar al caso como ejemplar, como prototipo idealizado. Otra reprobación que Dimock dirige a Freud consiste en el señalamiento de que el drama intrapsíquico del caso freudiano no deja lugar a la intersubjetividad en la forma de una historia familiar. En el relato psicoanlítico, Anna, aunque importante, es secundaria y prácticamente no entra en ni en la etiología del paciente ni en su desarrollo psíquico. Una consideración de la relación de Anna con el Hombre de los Lobos en la estructura de la dinámica de la familia queda fuera de consideración en el caso. Empero, el hecho de que Freud no explore las circunstancias por las cuales Anna a los cinco años de edad se involucre en una actividad sexual manifiesta en sus juegos de seducción con su hermano es claramente derivable de 1) su abandono de la teoría de la seducción -teoría fundamental en los ejemplares anteriores- en la que el abuso sexual de un niño por parte de un adulto constituye el corazón de la etiología de la histeria; 2) su nueva teoría de la sexualidad según la cual la misma no comienza en la pubertad sino que está presente desde el comienzo de la vida humana y fundamentalmente, 3) su concepción de patología psíquica según la cual la etiología debe ser completamente psíquica, es decir, causa y efecto deben pertenecer como hechos de la realidad psíquica. 
El ejemplar, como se sostuvo más arriba, encarna perspectivas metafísicas, valores, se estructura en un lenguaje teórico determinado y ésto es lo que debe examinarse en la consideración de un ejemplar. En conclusión, el caso permite hacer visible el complejo de Edipo, la sexualidad infantil y el hecho infantil en la situación inicial de la formación de la neurosis obsesiva, codeterminando los puntos en los que el individuo fracasa al enfrentar los problemas reales que la vida le presenta. También permite ver el significado crucial de la interpretación de los sueños en la práctica psicoanalítica. En los casos del apartado anterior, hay hechos que son externos a la vida psíquica del paciente y, en forma de recuerdo, actúan sobre la vida psíquica de éste. Aquí es lo inverso, es la realidad psíquica actuando sobre situaciones que el individuo vive.

\section{Conclusiones}

Si los ejemplares son modos estándares de resolver problemas, los primeramente presentados resuelven el problema de dar cuenta de los síntomas de modo totalmente diferente al del El Hombre de los Lobos.

Los síntomas se descifran y solucionan de modos diferentes. En los primeros ejemplares, mediante su eliminación. En el Hombre de los Lobos, trayendo a luz su significado. Allá, el síntoma no es visto como significando esa vivencia concreta y efectiva, sino como efecto de su representación. El síntoma es percibido como efecto de algo que ocurrió en el pasado. La situación es totalmente diferente en el conjunto de ejemplares correspondientes a la época psicoanalítica. El síntoma ya no es una consecuencia sino un significado - el significado de un conflicto. Por eso ya no se busca eliminarlo sino elucidarlo. Son dos prácticas diferentes, son dos modos de hacer diferentes. El relato del fenómeno onírico permite elucidar el síntoma, pues cuando se ve a un neurótico se ve la acción de fantasías en conflictos, algunas de las cuales se ligan al pasado del individuo y no representaciones de hechos que desde el pasado lo atormentan. Como los sueños son hechos de la misma materia que los síntomas, las conexiones oníricas que emergen en la traducción verbal nos sirven de datos para inteligir el mundo psíquico.

La escena primordial encarna la conceptualización del síntoma como la realización de una fantasía, realización que no exige una efectividad empírica. En el correlato empírico de la ontogénesis del paciente los momentos cronológicos sólo ganan relevancia si pueden ser asociados a una fantasía - no a una asociación causal. Dicha escena podría haber sido efectiva o no, lo decisivo es la fantasía que se une a ella. La cópula de los padres delante del niño puede haber sido algo que el niño observó o algo que imaginó y no interesa elegir entre estas dos opciones. Que esta escena sea parte de la anamnesis es, justamente, una prueba de que la visión se extiende mucho más allá de nuestras percepciones. El segundo grupo de ejemplares determina un dominio de aplicaciones en los que la similitud es generada por la estructura del complejo de Edipo. En el primer grupo, la semejanza es producida por la representación del hecho efectivo traumático. Esta diferencia entre ambas series implica diferencias de experiencias científicas a partir de los dominios modelados por las respectivas relaciones de similitud. 


\section{Notas e referências bibliográficas}

Leticia Minhot es Dra. en Filosofía, titulo otorgado por la Universidade Estadual de Campinas, Brasil. El título de grado; licenciada en Filosofía, lo obtuvo en la Universidad Nacional de Córdoba. En la actualidad es profesora titular en la Universidad Nacional de Córdoba. Es coeditora de la Revista Representaciones. Revista de Estudios sobre Arte, Ciencia y Filosofía. Sus áreas de investigación son la filosofía política de la ciencia, la epistemología, y la filosofía del psicoanálisis.

1 DIMOCK, G. Anna and the wolf-man: Rewriting Freud's case history. Representations, n. 50, p. 53-75, 1995.

2 REISCH, G. Did Kuhn kill logical empiricism? Philosophy of science, v. 58, n. 2, p. 265, 1991.

3 IRZIK, G.; GRÜNBERG, T. Carnap and Kuhn: Arch enemies or close allies? The British Journal for the Philosophy of Science, v. 46, n. 3, p. 285, 1995.

4 TAYLOR, C. Interpretation and the sciences of man. The Review of Metaphysics, v. 25, n. 1, p. 3-51, 1979. (Versão castelhana: La interpretación y las ciencias del hombre. In: . La libertad de los modernos. Buenos Aires: Amorrortu, 2005. p. 152-154).

$5 \quad$ ROUSE, J. Knowledge and power: Toward a political philosophy of science. Ithaca: Cornell University Press, 1987. p. 37.

6 SUPPE, F. (Ed.). The structure of scientific theories. Chicago, University of Illinois Press, 1974. (Versão castelhana: La estructura de las teorías científicas. UNED, Madrid, 1979. p. 195).

7 ROUSE, op.cit., p. 38-39.

8 KUHN, T. S. The structure of scientific revolutions. 2. ed. Chicago: University of Chicago Press, [1962/1970].(Versão castelhana: La estructura de las revoluciones científicas. México: F.C.E., 1975).

KUHN, T. S. (1970a), Postscript: 1969. In: _ _. The structure of scientific revolutions. Chicago: University of Chicago Press. 2. ed.. 1970a . (Versão castelhana: La estructura de las revoluciones cientificas. México: F.C.E., 1975).

$9 \quad$ NICKLES, Th. (2000) Kuhnian puzzle solving and schema theory. Philosophy of Science, v. 67, supplement. Proceedings of the 1998 Biennial Meetings of the Philosophy of Science Association. Part II: Symposia Papers, p. S242-S255. Chicago: The University of Chicago Press on behalf of the Philosophy of Science Association, 2000.

10 KUHN, T. (1977) The essential tension. Selected studies in scientific tradition and change. Chicago: University of Chicago, 1977. (Versão castelhana: Tensión essencial. México: F.C.E., 1996).

11 KUHN, Th. (2000). The road since structure Chicago: University of Chicago, 2000. (Edited by James Conant and John Haugeland) (Versão castelhana: EI camino desde la estructura. Barcelona: Paidós, 2002).

NICKLES, op. cit., 2000.

13 NICKLES, ibid.

14 BREUER, J.; FREUD, S. Estudios sobre la histeria. In: de: J. L. Etcheverry.

15 BREUER; FREUD, op. cit., 124-140.

16 BREUER; FREUD, op. cit., 141-150.

17 BREUER; FREUD, op. cit., 151-194.

18 BREUER; FREUD, op. cit.

19 El tratamiento de estos casos-patrón puede verse en MINHOT, L. La mirada psicoanalítica. Córdoba: Brujas, 2003 y en MINHOT, L. El psicoanálisis freudiano como actividad solucionadora de problemas. In: . Relendo a psicanálisis com Loparic. São Paulo: DWW Editorial, 2012.

20 El tratamiento de estos casos-patrón puede verse en Minhot (2003), Dora y el Hombre de los Lobos también en Minhot (2012)

21 FREUD, S. De la historia de una neurosis infantil. In: Obras completas. Buenos Aires: Amorrortu Editores, 1918. v. XVII, p. 110. Tradução de: J. L. Etcheverry.

22 FREUD, op. cit., p. 89.

23 FREUD, op. cit., p. 10

24 FREUD, op. cit., p. 52

25 FREUD, op. cit., p. 29.

26 FREUD, op. cit., p. 33

27 FREUD, S. La interpretación de los sueños. In: J. L.Etcheverry. . Obras completas. Buenos Aires: Amorrortu Editores, 1900. v. IV; V, 1994. p. 203. Tradução de:

28 FREUD, op. cit. 1918, p. 41, n. 17.

29 FREUD, op. cit. 1918, p. 100

30 DIMOCK, op. cit.

31 DIMOCK, op. cit.

[Recebido em Julho de 2013. Aprovado para publicação em Novembro de 2013] 